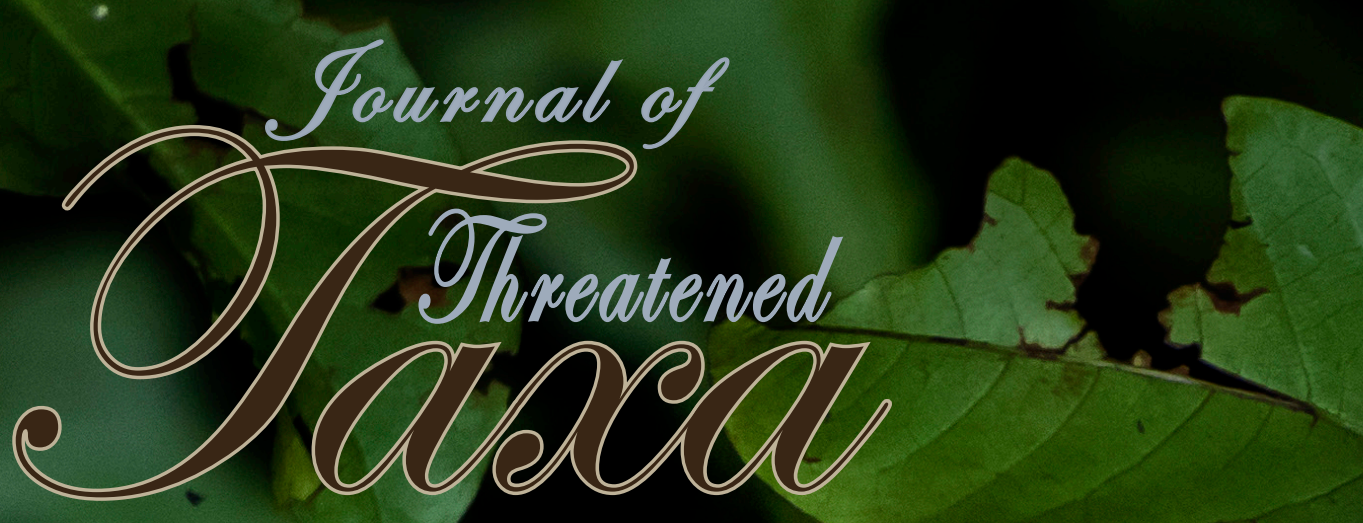

Building exidence for conservation glabally

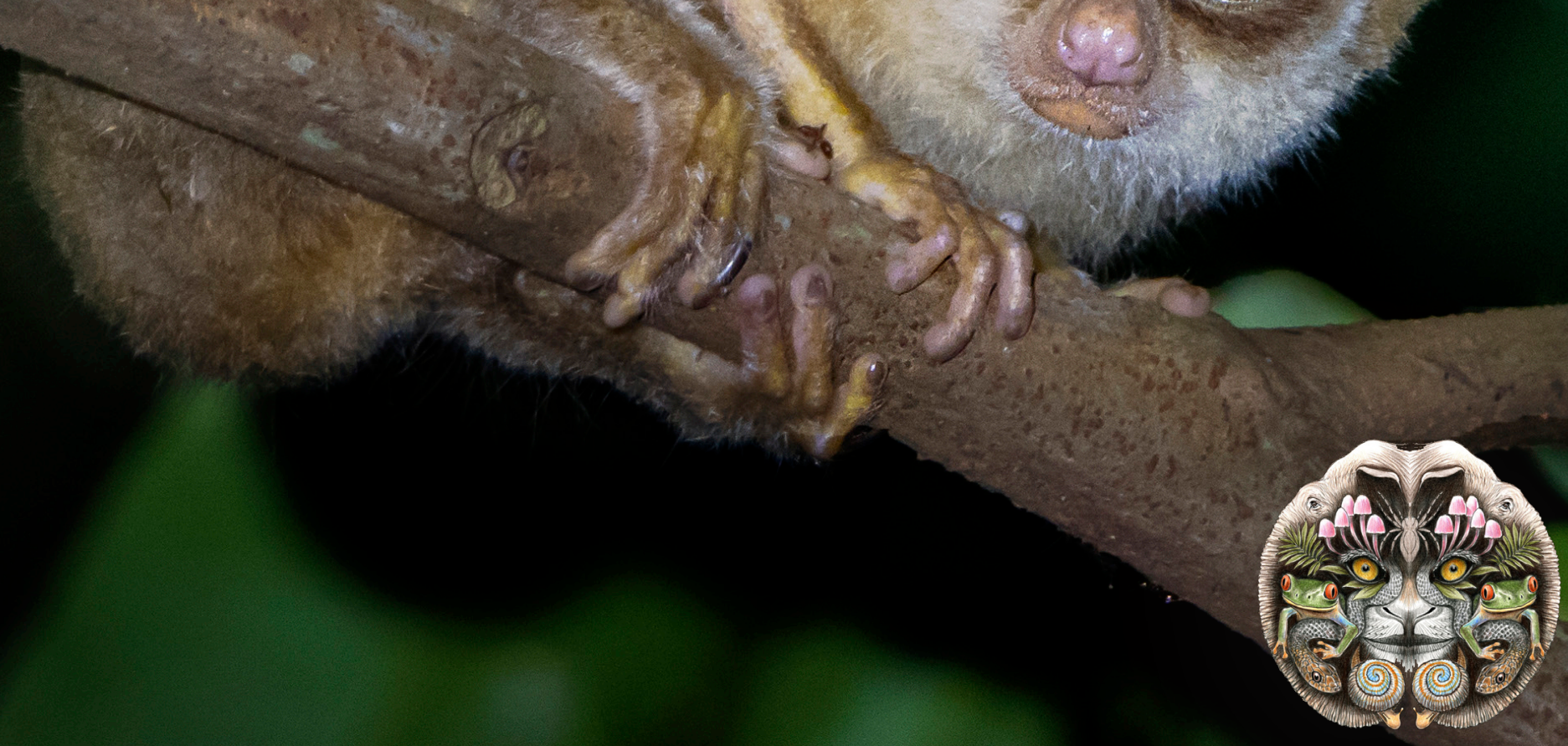

Open Access

$10.11609 /$ jott.2021.13.010.19431-19614 creven.threatenedtaxa.arg

26 September 2021 (Online \& Print) Val. 13 | Na. 11 | Pages: 19431-19674 


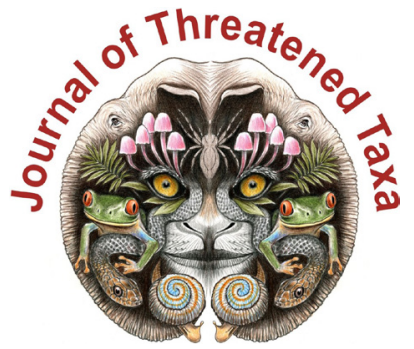

ISSN 0974-7907 (Online); ISSN $0974-7893$ (Print)

Publisher

Host

Wildlife Information Liaison Development Society

www.wild.zooreach.org

Zoo Outreach Organization www.zooreach.org

No. 12, Thiruvannamalai Nagar, Saravanampatti - Kalapatti Road, Saravanampatti, Coimbatore, Tamil Nadu 641035, India

Ph: +91 9385339863 | www.threatenedtaxa.org

Email: sanjay@threatenedtaxa.org

EDITORS

\section{Founder \& Chief Editor}

Dr. Sanjay Molur

Wildlife Information Liaison Development (WILD) Society \& Zoo Outreach Organization (ZOO)

12 Thiruvannamalai Nagar, Saravanampatti, Coimbatore, Tamil Nadu 641035, India

\section{Deputy Chief Edito}

Dr. Neelesh Dahanukar

Noida, Uttar Pradesh, India

\section{Managing Editor}

Mr. B. Ravichandran, WILD/ZOO, Coimbatore, India

\section{Associate Editors}

Dr. Mandar Paingankar, Government Science College Gadchiroli, Maharashtra 442605, India

Dr. Ulrike Streicher, Wildlife Veterinarian, Eugene, Oregon, USA

Ms. Priyanka Iyer, ZOO/WILD, Coimbatore, Tamil Nadu 641035, India

Dr. B. A. Daniel, $200 / \mathrm{WILD}$, Coimbatore, Tamil Nadu 641035, India

\section{Editorial Board}

Dr. Russel Mittermeier

Executive Vice Chair, Conservation International, Arlington, Virginia 22202, USA

\section{Prof. Mewa Singh Ph.D., FASc, FNA, FNASc, FNAPsy}

Ramanna Fellow and Life-Long Distinguished Professor, Biopsychology Laboratory, and Institute of Excellence, University of Mysore, Mysuru, Karnataka 570006, India; Honorary Professor, Jawaharlal Nehru Centre for Advanced Scientific Research, Bangalore; and Adjunct Professor, National Institute of Advanced Studies, Bangalore

\section{Stephen D. Nash}

Scientific Illustrator, Conservation International, Dept. of Anatomical Sciences, Health Sciences Center, T-8, Room 045, Stony Brook University, Stony Brook, NY 11794-8081, USA

\section{Dr. Fred Pluthero}

Toronto, Canada

\section{Dr. Priya Davidar}

Sigur Nature Trust, Chadapatti, Mavinhalla PO, Nilgiris, Tamil Nadu 643223, India

\section{Dr. Martin Fisher}

Senior Associate Professor, Battcock Centre for Experimental Astrophysics, Cavendish

Laboratory, JJ Thomson Avenue, Cambridge CB3 OHE, UK

\section{Dr. John Fellowe}

Honorary Assistant Professor, The Kadoorie Institute, 8/F, T.T. Tsui Building, The University of Hong Kong, Pokfulam Road, Hong Kong

\section{Prof. Dr. Mirco Solé}

Universidade Estadual de Santa Cruz, Departamento de Ciências Biológicas, Vice-coordenado do Programa de Pós-Graduação em Zoologia, Rodovia Ilhéus/Itabuna, Km 16 (45662-000)

Salobrinho, Ilhéus - Bahia - Brasil

\section{Dr. Rajeev Raghavan}

Professor of Taxonomy, Kerala University of Fisheries \& Ocean Studies, Kochi, Kerala, India

\section{English Editors}

Mrs. Mira Bhojwani, Pune, India

Dr. Fred Pluthero, Toronto, Canad

Mr. P. Ilangovan, Chennai, India

Web Maintenance

Mrs. Latha G. Ravikumar, ZOO/WILD, Coimbatore, India

\section{Typesetting}

Mr. Arul Jagadish, ZOO, Coimbatore, India

Mrs. Radhika, ZOO, Coimbatore, India

Mrs. Geetha, ZOO, Coimbatore India

\section{Fundraising/Communications}

Mrs. Payal B. Molur, Coimbatore, India

Subject Editors 2018-2020

Fungi

Dr. B. Shivaraju, Bengaluru, Karnataka, India

Dr. R.K. Verma, Tropical Forest Research Institute, Jabalpur, India

Dr. Vatsavaya S. Raju, Kakatiay University, Warangal, Andhra Pradesh, India

Dr. M. Krishnappa, Jnana Sahyadri, Kuvempu University, Shimoga, Karnataka, India

Dr. K.R. Sridhar, Mangalore University, Mangalagangotri, Mangalore, Karnataka, India

Dr. Gunjan Biswas, Vidyasagar University, Midnapore, West Bengal, India

Plants

Dr. G.P. Sinha, Botanical Survey of India, Allahabad, India

Dr. N.P. Balakrishnan, Ret. Joint Director, BSI, Coimbatore, India

Dr. Shonil Bhagwat, Open University and University of Oxford, UK

Prof. D.J. Bhat, Retd. Professor, Goa University, Goa, India

Dr. Ferdinando Boero, Università del Salento, Lecce, Italy

Dr. Dale R. Calder, Royal Ontaro Museum, Toronto, Ontario, Canada

Dr. Cleofas Cervancia, Univ. of Philippines Los Baños College Laguna, Philippines

Dr. F.B. Vincent Florens, University of Mauritius, Mauritius

Dr. Merlin Franco, Curtin University, Malaysia

Dr. V. Irudayaraj, St. Xavier's College, Palayamkottai, Tamil Nadu, India

Dr. B.S. Kholia, Botanical Survey of India, Gangtok, Sikkim, India

Dr. Pankaj Kumar, Kadoorie Farm and Botanic Garden Corporation, Hong Kong S.A.R., China

Dr. V. Sampath Kumar, Botanical Survey of India, Howrah, West Bengal, India

Dr. A.J. Solomon Raju, Andhra University, Visakhapatnam, India

Dr. Vijayasankar Raman, University of Mississippi, USA

Dr. B. Ravi Prasad Rao, Sri Krishnadevaraya University, Anantpur, India

Dr. K. Ravikumar, FRLHT, Bengaluru, Karnataka, India

Dr. Aparna Watve, Pune, Maharashtra, India

Dr. Qiang Liu, Xishuangbanna Tropical Botanical Garden, Yunnan, China

Dr. Noor Azhar Mohamed Shazili, Universiti Malaysia Terengganu, Kuala Terengganu, Malaysia Dr. M.K. Vasudeva Rao, Shiv Ranjani Housing Society, Pune, Maharashtra, India

Prof. A.J. Solomon Raju, Andhra University, Visakhapatnam, India

Dr. Mandar Datar, Agharkar Research Institute, Pune, Maharashtra, India

Dr. M.K. Janarthanam, Goa University, Goa, India

Dr. K. Karthigeyan, Botanical Survey of India, India

Dr. Errol Vela, University of Montpellier, Montpellier, France

Dr. P. Lakshminarasimhan, Botanical Survey of India, Howrah, India

Dr. Larry R. Noblick, Montgomery Botanical Center, Miami, USA

Dr. K. Haridasan, Pallavur, Palakkad District, Kerala, India

Dr. Analinda Manila-Fajard, University of the Philippines Los Banos, Laguna, Philippines

Dr. P.A. Sinu, Central University of Kerala, Kasaragod, Kerala, India

Dr. Afroz Alam, Banasthali Vidyapith (accredited A grade by NAAC), Rajasthan, India

Dr. K.P. Rajesh, Zamorin's Guruvayurappan College, GA College PO, Kozhikode, Kerala, India

Dr. David E. Boufford, Harvard University Herbaria, Cambridge, MA 02138-2020, USA

Dr. Ritesh Kumar Choudhary, Agharkar Research Institute, Pune, Maharashtra, India

Dr. Navendu Page, Wildlife Institute of India, Chandrabani, Dehradun, Uttarakhand, India

Invertebrates

Dr. R.K. Avasthi, Rohtak University, Haryana, India

Dr. D.B. Bastawade, Maharashtra, India

Dr. Partha Pratim Bhattacharjee, Tripura University, Suryamaninagar, India

Dr. Kailash Chandra, Zoological Survey of India, Jabalpur, Madhya Pradesh, India

Dr. Ansie Dippenaar-Schoeman, University of Pretoria, Queenswood, South Africa

Dr. Rory Dow, National Museum of natural History Naturalis, The Netherlands

Dr. Brian Fisher, California Academy of Sciences, USA

Dr. Richard Gallon, llandudno, North Wales, LL30 1UP

Dr. Hemant V. Ghate, Modern College, Pune, India

Dr. M. Monwar Hossain, Jahangirnagar University, Dhaka, Bangladesh

Mr. Jatishwor Singh Irungbam, Biology Centre CAS, Branišovská, Czech Republic

Dr. lan J. Kitching Natural History Museum, Cromwell Road, UK

Dr. George Mathew, Kerala Forest Research Institute, Peechi, India

Dr. John Noyes, Natural History Museum, London, UK

For Focus, Scope, Aims, and Policies, visit https://threatenedtaxa.org/index.php/JoTT/aims_scope
For Article Submission Guidelines, visit https://threatenedtaxa.org/index.php/JoTT/about/submissions
For Policies against Scientific Misconduct, visit https://threatenedtaxa.org/index.php/JoTT/policies_various 


\title{
Two new additions to the orchid flora of Assam, India
}

\author{
Sanswrang Basumatary ${ }^{1}$ (D), Sanjib Baruah ${ }^{2}$ iD $\&$ Lal Ji Singh ${ }^{3}$ (D)
}

\footnotetext{
1,2 Department of Botany, Bodoland University, Kokrajhar, Bodoland Territorial Region (BTR), Assam, 783370, India. ${ }^{3}$ Botanical Survey of India, Andaman \& Nicobar Regional Centre, Port Blair, Andaman \& Nicobar Islands 744102, India. ${ }^{1}$ basumatarysunlyte@gmail.com, ${ }^{2}$ sanjibbaruah9@gmail.com (corresponding author), ${ }^{3}$ laljisingh1970@rediffmail.com
}

Orchidaceae is one of the largest family and highly advanced monocotyledonous plants consisting of $\mathrm{c}$. 28,000 species under 736 genera in the world (Chase et al. 2015; Christenhusz \& Byng 2016). Bulbophyllum Thouars is one of the largest genera of Orchidaceae comprising c. 2000 species distributed in tropical and subtropical region of the world (Pearce \& Cribb 2002; Pridgeon et al. 2014; Averyanov et al. 2018). In India it is represented by 134 species, including one subspecies, and two varieties (Singh et al. 2019). In northeastern India the genus is represented by 75 species and three varieties (Rao 2007). Assam contains 35 species and two varieties (Gogoi 2017).

During a floristic survey in Ultapani Forest of Chirang Reserve Forest, Kokrajhar under the Manas Biosphere Reserve, Assam, some specimens of Bulbophyllum were collected. To verify the identity of these specimens, we undertook morphological comparisons to earlier collections based on online available herbarium specimens at L, K, AMES, NY, P and consulting relevant literature (Averyanov \& Averyanova 2003; Vermeulen \& Byrne 2011; Wood et al. 2011; Averyanov 2013; Li et al. 2013; Vermeulen et al. 2015; Averyanov et al. 2016).

After critical examination these specimens were found to represent $B$. tenuifolium (Blume) Lindl. and $B$. parviflorum C.S.P. Parish \& Rchb.f. which are hitherto unrecorded for Assam state (Bose \& Bhattacharjee 1980; Sarkar 1995; Misra 2007; Rao 2007; Gogoi 2017; Mao \& Deori 2018; Singh et al. 2019; Singh \& Ranjan 2021) and are therefore reported here as new records to the flora of Assam. Of them, B. tenuifolium was earlier recorded from Andaman \& Nicobar Islands by Kumar \& Sreekumar (2002).

Representative specimens of the species are deposited in Herbarium of Botanical Survey of India (BSI), Andaman \& Nicobar Regional Centre Herbarium (PBL) and Bodoland University Botanical Herbarium $(\mathrm{BUBH})$, Kokrajhar, Assam. Field photographs of the species are provided for easy identification.

\section{Bulbophyllum tenuifolium (Blume) Lindl. (Figure 1 \& Image 1)}

Gen. Sp. Orchid. PI.: 50 (1830); Diphyes tenuifolia Blume, Bijdr. Fl. Ned. Ind.: 316 (1825). Phyllorkis tenuifolia (Blume) Kuntze, Revis. Gen. PI. 2: 678 (1891). Bulbophyllum angulatum J.J.Sm., Bull. Dép. Agric. Indes Néerl. 15: 19 (1908). Bulbophyllum microstele

Editor: Pankaj Kumar, Kadoorie Farm and Botanic Garden (KFBG) Corporation, Tai Po, Hong Kong S.A.R., China. Date of publication: 26 September 2021 (online \& print) Citation: Basumatary, S., S. Baruah \& L.J. Singh (2021). Two new additions to the orchid flora of Assam, India. Journal of Threatened Taxa 13(11): $19665-19670$. https://doi.org/10.11609/jott.7282.13.11.19665-19670

Copyright: (c) Basumatary et al. 2021. Creative Commons Attribution 4.0 International License. JoTT allows unrestricted use, reproduction, and distribution of this article in any medium by providing adequate credit to the author(s) and the source of publication.

Funding: Self-funded.

Competing interests: The authors declare no competing interests.

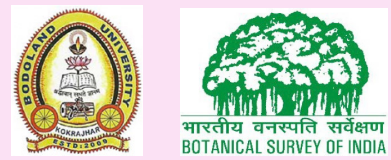

Acknowledgements: Authors are thankful to Dr. A. A. Mao, Director, Botanical Survey of India, Kolkata and to Dr. Rebecca Daimari, HOD, Dept of Botany, Bodoland University, Kokrajhar, Assam for all kinds of support. The first author is indebted to Bana Kumar Brahma, a dedicated worker of 'Biodiversity Conservation Society' an NGO situated at Ultapani Forest Range, Kokrajhar, Assam for his active participation during the field visit. 


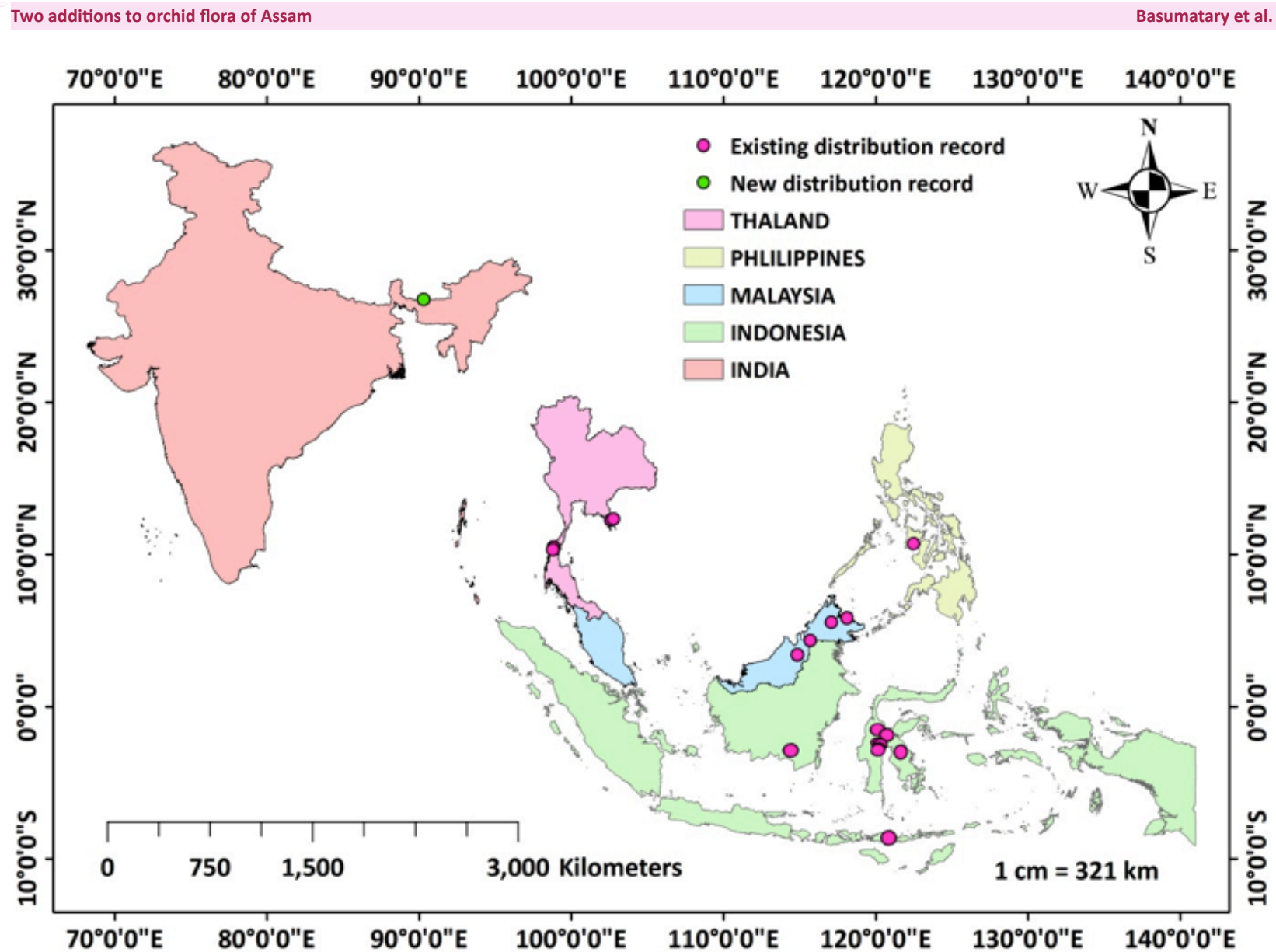

Figure 1. Distribution of Bulbophyllum tenuifolium (Blume) Lindl. across the world (Data shows as per GBIF).

Schltr., Repert. Spec. Nov. Regni Veg. 8: 569 (1910). Cirrhopetalum chryseum Kraenzl., Repert. Spec. Nov. Regni Veg. 8: 97 (1910); Bulbophyllum chryseum (Kraenzl.) Ames, Philipp. J. Sci., C 6: 54 (1911). Bulbophyllum nigromaculatum Holttum, Gard. Bull. Singapore 11: 276 (1947). Bulbophyllum konstantinovii Aver., Turczaninowia 16(4): 29 (2013).

Type: Indonesia: Java, Salak (?), coll. Blume 639 (L, holotype HLB 902.322479). Malaysia: Sarawak, Bei Kutching, 13.xii.1926, coll. Schlechter 15835 (K!, isotype [K000829845]).

Description: Dwarf creeping epiphyte, rhizome wiry, thin, greenish on young, later whitish-grey, 0.6-1 $\mathrm{mm}$ in diameter, pseudobulbs $0.7-2.2 \mathrm{~cm}$ apart from each other; green to yellowish-green, ovate, 5-10 mm tall, 2-6 $\mathrm{mm}$ in diameter, oblique in slightly bending to rhizome, longitudinally irregularly grooved with single apical leaf; leaves leathery, narrowly ovate, $1.5-5 \times$ $0.4-1 \mathrm{~cm}$, apex obtuse, petiole very short or subsessile; inflorescence arising from the base of pseudobulb, sometimes from the matured rhizome, $1.5-3 \mathrm{~cm}$ long, with single terminal flower, ascending, filiform, glabrous, light yellowish-green; stalk $1-2 \mathrm{~cm}$ long with small bract at the base; bracts $0.5-1 \mathrm{~mm}$ long, 0.2-0.3 $\mathrm{mm}$ in diameter; pedicel 6-10 $\mathrm{mm}$ long, filiform; flowers $1 \mathrm{~cm}$ across, with spreading lateral sepals; sepals light dull-yellowish with purple brown stripes, 4-6 × 1-2 mm, three distinct nerves, apex acute; median sepal elliptic with more darker stripes; lateral sepals narrowly ovate, spreading, slightly longer than the median sepals, slightly oblique at base; petals oblique ovate, 1.5-2 × 1-1.3 mm, bright-yellow, apex acute, margin with irregular dark purple spots; lip simple, elliptic 3-5 × 1-1.5 mm, yellow, base narrowing, forming bending neck, jointed with column foot apex; column erect, c. $0.8 \times 0.5 \mathrm{~mm}$, brightyellow, apex with 2 straight, c. $0.6 \mathrm{~mm}$ long stelidia; column head broadening into cup-shaped, c. $1 \times 1 \mathrm{~mm}$, operculum concave, ovoid, c. $0.4 \mathrm{~mm}$, yellow; pollinia 2, globose, yellow.

Flowering \& fruiting: November-January.

Distribution: India (Andaman \& Nicobar Islands, Assam), Borneo, Cambodia, Java, Lesser Sunda Island, Malaysia, Philippines, Sulawesi, Sumatra, Thailand.

Habitat \& ecology: Epiphytic on small branches of 

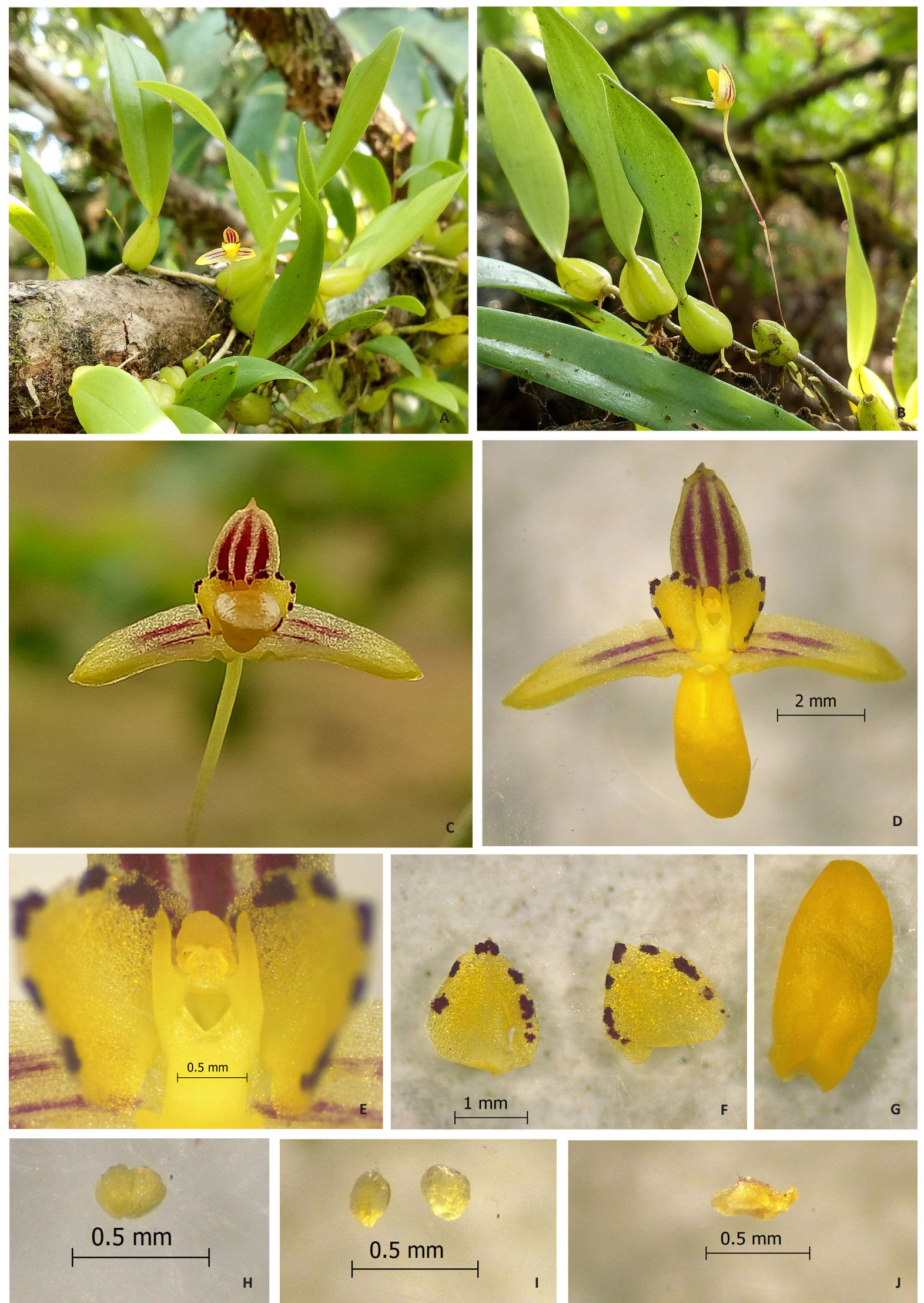

Image 1. Bulbophyllum tenuifolium (Blume) Lindl.: A \& B-Habit | C \& D-Flowers | E-Showing stelidia | F-Petals | G-Lip | H \& I-Pollinia | J-Anther cap. @ Sanswrang Basumatary. 
trees in evergreen or semi-evergreen humid forest along a stream at elevations of 100-700 m.

Specimens examined: India: Assam, Chirang Reserve Forest, Ultapani, 197m, 18.i.2021, coll. Sanswrang Basumatary \& Sanjib Baruah, 0268 (BUBH, acc. no. 0000411). Andaman \& Nicobar Islands, Middle Andaman, Kadamtala Reserve Forest, 01.xi. 2012, coll. Lal Ji Singh, 29572 (PBL, acc. no. 38319); Little Andaman, Krishna Nallah, 13.x. 2015, coll. Lal Ji Singh, 29673 (PBL, acc. no. 38320). Philippines: Leyte, Panda, Dagami, 11.v.1913, coll. C.A. Wenzel, 93 (NY, 04012457), (AMES, 00000415).

\section{Bulbophyllum parviflorum C.S.P. Parish \& Rchb.f. (Figure 2 \& Image 2)}

Trans. Linn. Soc. London 30: 152 (1874); Phyllorkis parviflora (C.S.P.Parish \& Rchb.f.) Kuntze, Revis. Gen. PI. 2: 677 (1891). Phyllorkis thomsonii (Hook.f.) Kuntze, Revis. Gen. PI. 2: 677 (1891); Bulbophyllum thomsonii Hook.f., Fl. Brit. India 6: 764 (1894).

Type: Myanmar: Tenasserim, coll. Parish 305 (W, holotype Herb No. 2273; K!, isotype [K000829138]).

Description: Rhizomes branched, pseudobulb compressed globose, with apical point, 1-1.5 cm in diameter, $3.5-7.5 \mathrm{~cm}$ apart; petiole up to $2.5 \mathrm{~cm}$ long; inflorescence arising from the base of mature pseudobulb, up to $20 \mathrm{~cm}$ long, many flowered; flower c. $4 \mathrm{~mm}$ in diameter, pedicels $2-4 \mathrm{~mm}$ long, green; bracts (found on peduncle) c. $8 \mathrm{~mm}$ long, c. $3 \mathrm{~mm}$ diameter, encircled the peduncle, brown, apex acute; bracts (found at the base of pedicel) $2.5-4 \mathrm{~mm}$ long, ovate-lanceolate, apex acute-acuminate, glabrous; sepals pubescent at margin, c. $4 \mathrm{~mm}$ long, c. $1.5 \mathrm{~mm}$ at base, median sepal c. $2.5 \mathrm{~mm}$ long, c. $1 \mathrm{~mm}$ in diameter at base; petals c. $2 \mathrm{~mm}$ long, c. $1.5 \mathrm{~mm}$ in diameter, margin pubescent, white, lip c. $3 \mathrm{~mm}$; anther cap c. $0.4 \mathrm{~mm}$, brownish; pollinia 2, c. $0.3 \mathrm{~mm}$, yellow.

Flowering \& fruiting: November-January.

Distribution: India (Arunachal Pradesh, Assam, Manipur, Mizoram, Sikkim, West Bengal), Bhutan, Cambodia, China, Laos, Myanmar, Thailand, Vietnam.

Habitat \& ecology: Epiphyte on branches of trees in semi-evergreen humid forest along a stream over tiny stones bedrock at elevations of 100-350 m.

Specimens examined: India: Assam: Chirang Reserve Forest, Ultapani, 215 m, 11.i.2021, coll. Sanswrang Basumatary \& Sanjib Baruah 0268 (BUBH, acc.no. 0000405). Sikkim, 1850, coll. Thomson s.n. (K, K000829139). Sikkim, 3000 ft, ix.1898, coll. Pantling 245 (P, P00362005), (L, L. 1488763).

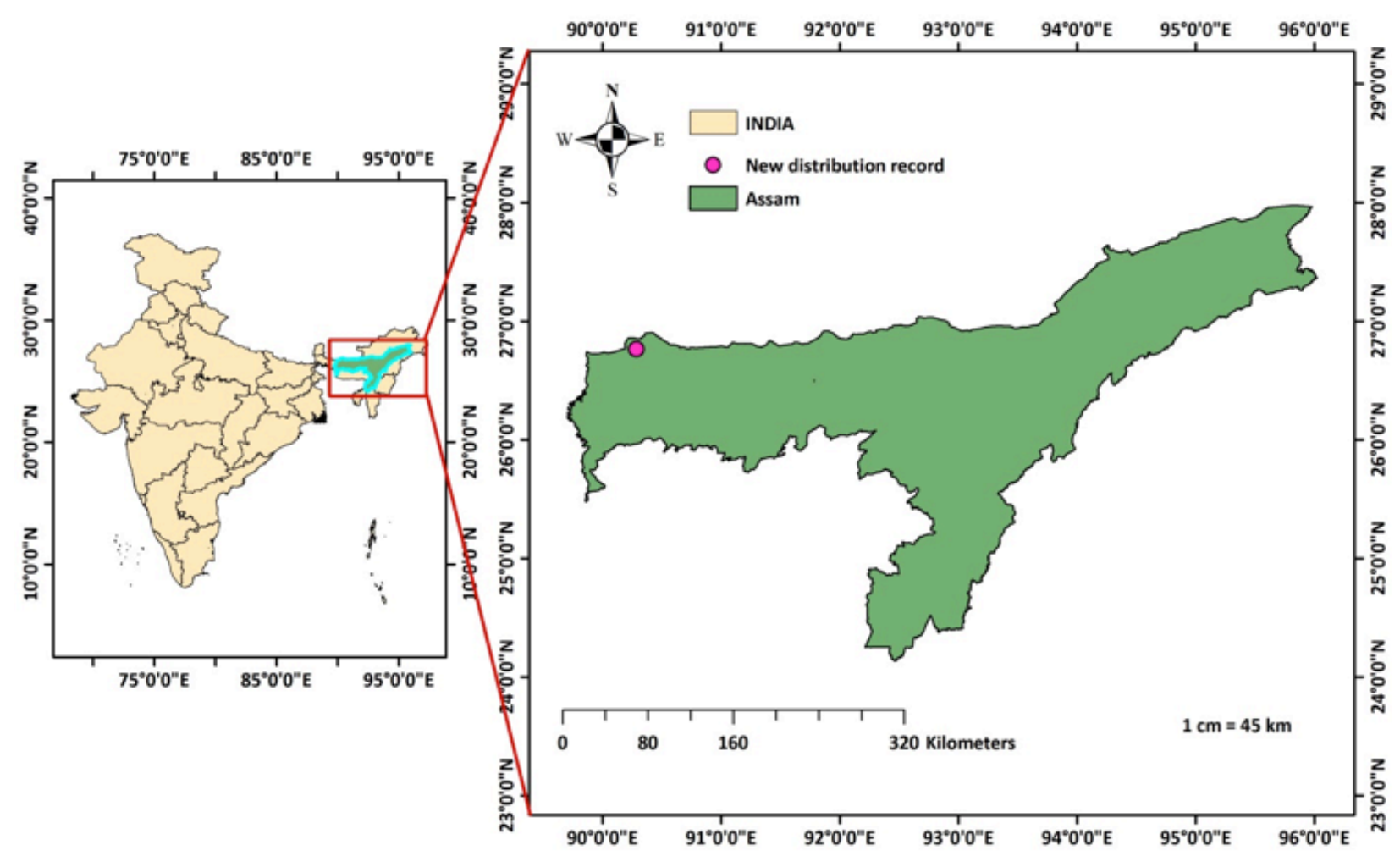

Figure 2. Distribution of Bulbophyllum parviflorum C.S.P. Parish \& Rchb.f. in Assam. 

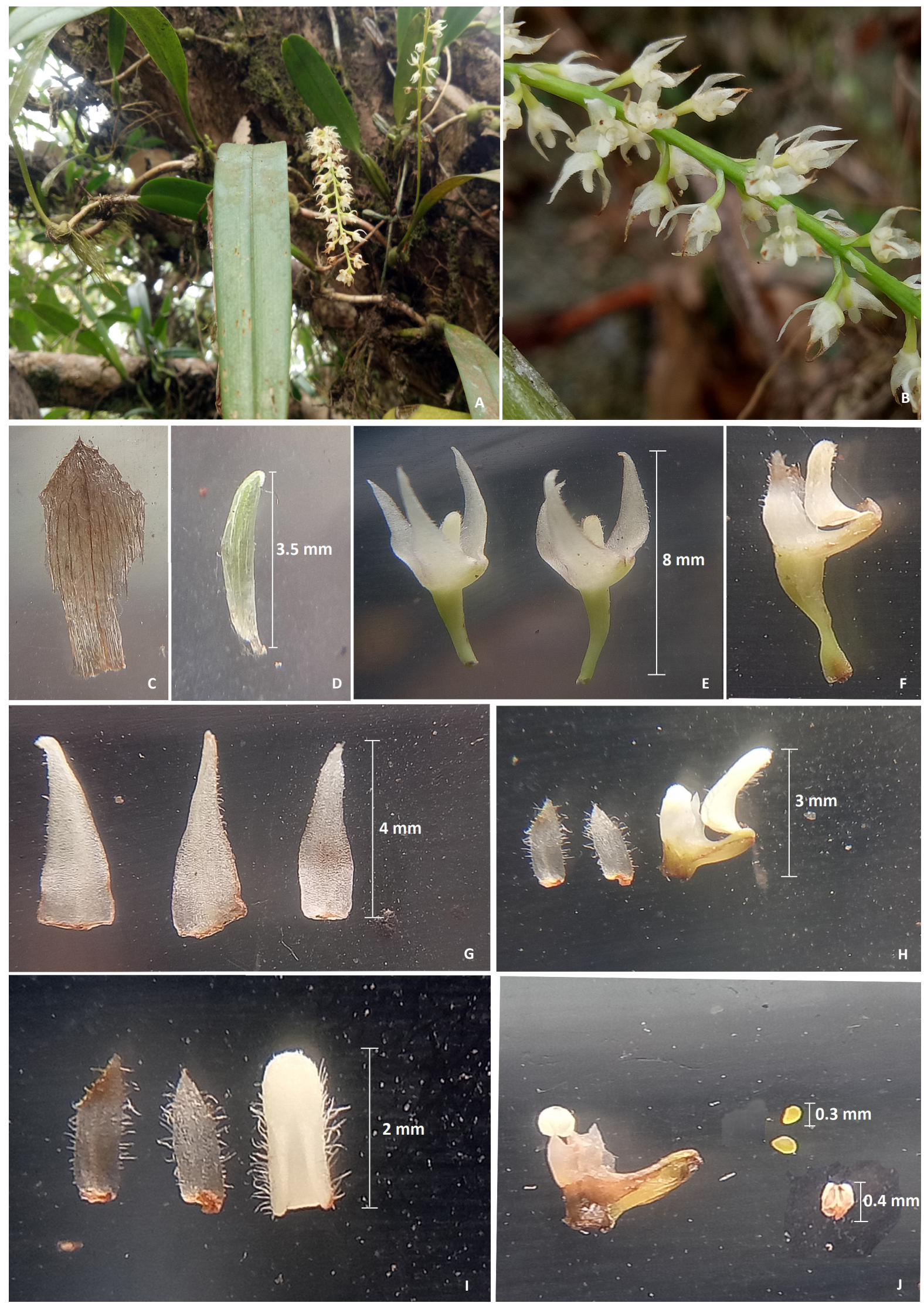

Image 2. Bulbophyllum parviflorum C.S.P. Parish \& Rchb.f.: A-Habit | B -Inflorescence | C \& D-Bracts | E-Flowers | F-Flower after removing sepals | G-Sepals | H \& I-Petals and lip | J-Stelidia, Pollen and Anther cap. OSanswrang Basumatary. 


\section{References}

Averyanov, L.V., N.V. Duy, N.H. Tuan, M.S. Nuraliev, T.V. Maisak \& N.C. Anh (2018). New species of Bulbophyllum (Orchidaceae) in the flora of Vietnam. Phytotaxa 369(1): 001-014. https://doi.org/10.11646/ phytotaxa.369.1.1

Averyanov, L.V. \& A.L. Averyanova (2003). Updated Checklist of the Orchids of Vietnam. Vietnam National University Publishing House, Hanoi, $101 \mathrm{pp}$.

Averyanov, L.V. (2013). New and rare orchids (Orchidaceae) in the flora of Cambodia and Laos. Turczaninowia 16(4): 26-46.

Averyanov, L.V., K.S. Nguyen, T.V. Maisak, E.L. Konstantinov, T.H. Nguyen \& S. Bounphanmy (2016). New and rare orchids (Orchidaceae) in the flora of Cambodia and Laos. Turczaninowia 19 $5-58$.

Bose, T.K \& S.K. Bhattacharjee (1980). Orchids of India. NayaProkash, Calcutta, $538 \mathrm{pp}$.

Chase, M.W., K.M. Cameron, J.V. Freudenstein, A.M. Pridgeon, G. Salazar, C. Van den Berg \& A. Schuiteman (2015). An updated classification of Orchidaceae. Botanical Journal of the Linnean Society 177: 151-174. https://doi.org/10.1111/boj.12234

Christenhusz, M.J.M. \& J.W. Byng (2016).The number of known plants species in the world and its annual increase. Phytotaxa 261(3): 201217. https://doi.org/10.11646/phytotaxa.261.3.1

Gogoi, K. (2017). Wild Orchids of Assam-A pictorial guide.Assam State Biodiversity Board, Guwahati, 380pp.

Kumar, V.S. \& P.V.Sreekumar (2002). On the Orchid, Bulbophyllum tenuifolium from the Kalpong Hydroelectric Project site, Andamans. Indian Forester 128: 81-83.
Li, J.W., D.P. Ye, Q. Liu \& J.T. Yin (2013). Two new records of orchid from China. Plant Diversity and Resources 35: 128-130.

Mao, A.A \& C. Deori (2018). Checklist of Orchids of Manipur- A Pictorial Handbook. Forest Department, Government of Manipur and Botanical Survey of India, 287pp.

Misra, S. (2007). Orchids of India - A Glimpse. Bishen Singh Mahendra Pal Singh, Dehradun, 402pp.

Pearce, N.R. \& P.J. Cribb (2002). The Orchids of Bhutan. The Charlesworth Group, Huddersfield, 643pp.

Pridgeon, A.M., P.J. Cribb, M.W. Chase \& F.N. Rasmussen (2014). Genera Orchidacearum, Vol. 6, Epidendroideae (part III). Oxford University Press, Oxford, 544pp.

Rao, A.N. (2007). Orchid Flora of north East India-An Update Analysis. Bulletin of Arunachal Forest Research 23: 6-38.

Sarkar, P.K. (1995). An up-to-date census of Indian orchids. Journal of Economic and Taxonomic Botany Additional Series 11: 1-31.

Singh, L.J. \& V. Ranjan (2021). New Vistas in Indian Flora. Vol. 1 \& 2 Bishen Singh Mahendra Pal Singh, Dehradun, Uttarakhand, India, 417pp \& 819pp.

Singh, S.K., D.K. Agarwala, J.S. Jalal, A.A. Mao \& P. Singh (2019) Orchids of India - A Pictorial Guide. Botanical Survey of India, Kolkata, 548pp.

Vermeulen, J. \& P. O’Byrne (2011). Bulbophyllum of Sulawesi. Natural History Publications (Borneo), Kota Kinabalu, Malaysia, 247pp.

Vermeulen, J., P. O’Byrne \& A. Lamb (2015). Bulbophyllum of Borneo. Nautural History Publications (Borneo), Kota Kinabalu, 728pp.

Wood, J.J., T.E. Beaman, A. Lamb, C.C. Lun \& J.H. Beaman (2011). The Orchids of Mount Kinabalu 2. Natural history publications (Borneo), Kota Kinabalu, Malaysia, 726pp. 
Dr. Albert G. Orr, Griffith University, Nathan, Australia

Dr. Sameer Padhye, Katholieke Universiteit Leuven, Belgium

Dr. Nancy van der Poorten, Toronto, Canada

Dr. Kareen Schnabel, NIWA, Wellington, New Zealand

Dr. R.M. Sharma, (Retd.) Scientist, Zoological Survey of India, Pune, India

Dr. Manju Siliwal, WILD, Coimbatore, Tamil Nadu, India

Dr. G.P. Sinha, Botanical Survey of India, Allahabad, India

Dr. K.A. Subramanian, Zoological Survey of India, New Alipore, Kolkata, India

Dr. P.M. Sureshan, Zoological Survey of India, Kozhikode, Kerala, India

Dr. R. Varatharajan, Manipur University, Imphal, Manipur, India

Dr. Eduard Vives, Museu de Ciències Naturals de Barcelona, Terrassa, Spain

Dr. James Young, Hong Kong Lepidopterists' Society, Hong Kong

Dr. R. Sundararaj, Institute of Wood Science \& Technology, Bengaluru, India

Dr. M. Nithyanandan, Environmental Department, La Ala Al Kuwait Real Estate. Co. K.S.C., Kuwait

Dr. Himender Bharti, Punjabi University, Punjab, India

Mr. Purnendu Roy, London, UK

Dr. Saito Motoki, The Butterfly Society of Japan, Tokyo, Japan

Dr. Sanjay Sondhi, TITLI TRUST, Kalpavriksh, Dehradun, India

Dr. Nguyen Thi Phuong Lien, Vietnam Academy of Science and Technology, Hanoi, Vietnam

Dr. Nitin Kulkarni, Tropical Research Institute, Jabalpur, India

Dr. Robin Wen Jiang Ngiam, National Parks Board, Singapore

Dr. Lional Monod, Natural History Museum of Geneva, Genève, Switzerland.

Dr. Asheesh Shivam, Nehru Gram Bharti University, Allahabad, India

Dr. Rosana Moreira da Rocha, Universidade Federal do Paraná, Curitiba, Brasil

Dr. Kurt R. Arnold, North Dakota State University, Saxony, Germany

Dr. James M. Carpenter, American Museum of Natural History, New York, USA

Dr. David M. Claborn, Missouri State University, Springfield, USA

Dr. Kareen Schnabel, Marine Biologist, Wellington, New Zealand

Dr. Amazonas Chagas Júnior, Universidade Federal de Mato Grosso, Cuiabá, Brasil

Mr. Monsoon Jyoti Gogoi, Assam University, Silchar, Assam, India

Dr. Heo Chong Chin, Universiti Teknologi MARA (UiTM), Selangor, Malaysia

Dr. R.J. Shiel, University of Adelaide, SA 5005, Australia

Dr. Siddharth Kulkarni, The George Washington University, Washington, USA

Dr. Priyadarsanan Dharma Rajan, ATREE, Bengaluru, India

Dr. Phil Alderslade, CSIRO Marine And Atmospheric Research, Hobart, Australia

Dr. John E.N. Veron, Coral Reef Research, Townsville, Australia

Dr. Daniel Whitmore, State Museum of Natural History Stuttgart, Rosenstein, Germany.

Dr. Yu-Feng Hsu, National Taiwan Normal University, Taipei City, Taiwan

Dr. Keith V. Wolfe, Antioch, California, USA

Dr. Siddharth Kulkarni, The Hormiga Lab, The George Washington University, Washington,

D.C., USA

Dr. Tomas Ditrich, Faculty of Education, University of South Bohemia in Ceske

Budejovice, Czech Republic

Dr. Mihaly Foldvari, Natural History Museum, University of Oslo, Norway

Dr. V.P. Uniyal, Wildlife Institute of India, Dehradun, Uttarakhand 248001, India

Dr. John T.D. Caleb, Zoological Survey of India, Kolkata, West Bengal, India

Dr. Priyadarsanan Dharma Rajan, Ashoka Trust for Research in Ecology and the Environment (ATREE), Royal Enclave, Bangalore, Karnataka, India

\section{Fishes}

Dr. Neelesh Dahanukar, IISER, Pune, Maharashtra, India

Dr. Topiltzin Contreras MacBeath, Universidad Autónoma del estado de Morelos, México

Dr. Heok Hee Ng, National University of Singapore, Science Drive, Singapore

Dr. Rajeev Raghavan, St. Albert's College, Kochi, Kerala, India

Dr. Robert D. Sluka, Chiltern Gateway Project, A Rocha UK, Southall, Middlesex, UK

Dr. E. Vivekanandan, Central Marine Fisheries Research Institute, Chennai, India

Dr. Davor Zanella, University of Zagreb, Zagreb, Croatia

Dr. A. Biju Kumar, University of Kerala, Thiruvananthapuram, Kerala, India

Dr. Akhilesh K.V., ICAR-Central Marine Fisheries Research Institute, Mumbai Research

Centre, Mumbai, Maharashtra, India

Dr. J.A. Johnson, Wildlife Institute of India, Dehradun, Uttarakhand, India

\section{Amphibians}

Dr. Sushil K. Dutta, Indian Institute of Science, Bengaluru, Karnataka, India

Dr. Annemarie Ohler, Muséum national d'Histoire naturelle, Paris, France

\section{Reptiles}

Dr. Gernot Vogel, Heidelberg, Germany

Dr. Raju Vyas, Vadodara, Gujarat, India

Dr. Pritpal S. Soorae, Environment Agency, Abu Dubai, UAE.

Prof. Dr. Wayne J. Fuller, Near East University, Mersin, Turkey

Prof. Chandrashekher U. Rivonker, Goa University, Taleigao Plateau, Goa. India

Dr. S.R. Ganesh, Chennai Snake Park, Chennai, Tamil Nadu, India

Dr. Himansu Sekhar Das, Terrestrial \& Marine Biodiversity, Abu Dhabi, UAE
Birds

Dr. Hem Sagar Baral, Charles Sturt University, NSW Australia

Dr. Chris Bowden, Royal Society for the Protection of Birds, Sandy, UK

Dr. Priya Davidar, Pondicherry University, Kalapet, Puducherry, India

Dr. J.W. Duckworth, IUCN SSC, Bath, UK

Dr. Rajah Jayapal, SACON, Coimbatore, Tamil Nadu, India

Dr. Rajiv S. Kalsi, M.L.N. College, Yamuna Nagar, Haryana, India

Dr. V. Santharam, Rishi Valley Education Centre, Chittoor Dt., Andhra Pradesh, India

Dr. S. Balachandran, Bombay Natural History Society, Mumbai, India

Mr. J. Praveen, Bengaluru, India

Dr. C. Srinivasulu, Osmania University, Hyderabad, India

Dr. K.S. Gopi Sundar, International Crane Foundation, Baraboo, USA

Dr. Gombobaatar Sundev, Professor of Ornithology, Ulaanbaatar, Mongolia

Prof. Reuven Yosef, International Birding \& Research Centre, Eilat, Israel

Dr. Taej Mundkur, Wetlands International, Wageningen, The Netherlands

Dr. Carol Inskipp, Bishop Auckland Co., Durham, UK

Dr. Tim Inskipp, Bishop Auckland Co, Durham, UK

Dr. V. Gokula, National College, Tiruchirappalli, Tamil Nadu, India

Dr. Arkady Lelej, Russian Academy of Sciences, Vladivostok, Russia

Dr. Simon Dowell, Science Director, Chester Zoo, UK

Dr. Mário Gabriel Santiago dos Santos, Universidade de Trás-os-Montes e Alto Douro,

Quinta de Prados, Vila Real, Portugal

Dr. Grant Connette, Smithsonian Institution, Royal, VA, USA

Dr. M. Zafar-ul Islam, Prince Saud Al Faisal Wildlife Research Center, Taif, Saudi Arabia

Mammals

Dr. Giovanni Amori, CNR - Institute of Ecosystem Studies, Rome, Italy

Dr. Anwaruddin Chowdhury, Guwahati, India

Dr. David Mallon, Zoological Society of London, UK

Dr. Shomita Mukherjee, SACON, Coimbatore, Tamil Nadu, India

Dr. Angie Appel, Wild Cat Network, Germany

Dr. P.O. Nameer, Kerala Agricultural University, Thrissur, Kerala, India

Dr. Ian Redmond, UNEP Convention on Migratory Species, Lansdown, UK

Dr. Heidi S. Riddle, Riddle's Elephant and Wildlife Sanctuary, Arkansas, USA

Dr. Karin Schwartz, George Mason University, Fairfax, Virginia.

Dr. Lala A.K. Singh, Bhubaneswar, Orissa, India

Dr. Mewa Singh, Mysore University, Mysore, India

Dr. Paul Racey, University of Exeter, Devon, UK

Dr. Honnavalli N. Kumara, SACON, Anaikatty P.O., Coimbatore, Tamil Nadu, India

Dr. Nishith Dharaiya, HNG University, Patan, Gujarat, India

Dr. Spartaco Gippoliti, Socio Onorario Società Italiana per la Storia della Fauna "Giuseppe

Altobello", Rome, Italy

Dr. Justus Joshua, Green Future Foundation, Tiruchirapalli, Tamil Nadu, India

Dr. H. Raghuram, The American College, Madurai, Tamil Nadu, India

Dr. Paul Bates, Harison Institute, Kent, UK

Dr. Jim Sanderson, Small Wild Cat Conservation Foundation, Hartford, USA

Dr. Dan Challender, University of Kent, Canterbury, UK

Dr. David Mallon, Manchester Metropolitan University, Derbyshire, UK

Dr. Brian L. Cypher, California State University-Stanislaus, Bakersfield, CA

Dr. S.S. Talmale, Zoological Survey of India, Pune, Maharashtra, India

Prof. Karan Bahadur Shah, Budhanilakantha Municipality, Kathmandu, Nepal

Dr. Susan Cheyne, Borneo Nature Foundation International, Palangkaraja, Indonesia

Dr. Hemanta Kafley, Wildlife Sciences, Tarleton State University, Texas, USA

\section{Other Disciplines}

Dr. Aniruddha Belsare, Columbia MO 65203, USA (Veterinary)

Dr. Mandar S. Paingankar, University of Pune, Pune, Maharashtra, India (Molecular)

Dr. Jack Tordoff, Critical Ecosystem Partnership Fund, Arlington, USA (Communities)

Dr. Ulrike Streicher, University of Oregon, Eugene, USA (Veterinary)

Dr. Hari Balasubramanian, EcoAdvisors, Nova Scotia, Canada (Communities)

Dr. Rayanna Hellem Santos Bezerra, Universidade Federal de Sergipe, São Cristóvão, Brazil

Dr. Jamie R. Wood, Landcare Research, Canterbury, New Zealand

Dr. Wendy Collinson-Jonker, Endangered Wildlife Trust, Gauteng, South Africa

Dr. Rajeshkumar G. Jani, Anand Agricultural University, Anand, Gujarat, India

Dr. O.N. Tiwari, Senior Scientist, ICAR-Indian Agricultural Research Institute (IARI), New

Delhi, India

Dr. L.D. Singla, Guru Angad Dev Veterinary and Animal Sciences University, Ludhiana, India

Dr. Rupika S. Rajakaruna, University of Peradeniya, Peradeniya, Sri Lanka

Dr. Bahar Baviskar, Wild-CER, Nagpur, Maharashtra 440013, India

Reviewers 2018-2020

Due to pausity of space, the list of reviewers for 2018-2020 is available online.
The opinions expressed by the authors do not reflect the views of the Journal of Threatened Taxa, Wildlife Information Liaison Development Society, Zoo Outreach Organization, or any of the partners. The journal, the publisher, the host, and the partners are not responsible for the accuracy of the political boundaries shown in the maps by the authors.

\footnotetext{
Print copies of the Journal are available at cost. Write to:

The Managing Editor, JoTT,

c/o Wildlife Information Liaison Development Society,

No. 12, Thiruvannamalai Nagar, Saravanampatti - Kalapatti Road,

Saravanampatti, Coimbatore, Tamil Nadu 641035, India

ravi@threatenedtaxa.org
} 


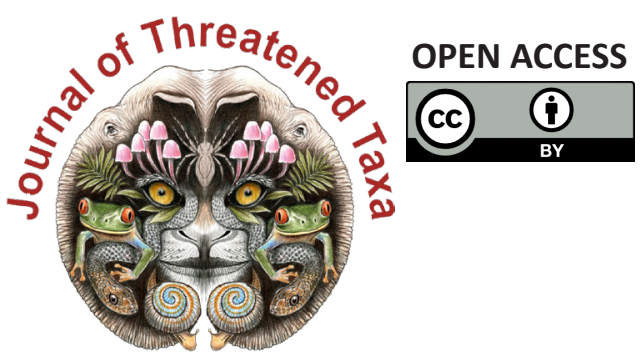

www.threatenedtaxa.org

The Journal of Threatened Taxa (JoTT) is dedicated to building evidence for conservation globally by publishing peer-reviewed articles online every month at a reasonably rapid rate at www.threatenedtaxa.org. All articles published in JoTT are registered under Creative Commons Attribution 4.0 International License unless otherwise mentioned. JoTT allows allows unrestricted use, reproduction, and distribution of articles in any medium by providing adequate credit to the author(s) and the source of publication.

\section{ISSN $0974-7907$ (Online) | ISSN $0974-7893$ (Print)}

\section{September 2021 | Vol. 13 | No. 11 | Pages: 19431-19674 Date of Publication: 26 September 2021 (Online \& Print) DOI: 10.11609/jott.2021.13.11.19431-19674}

Articles

Understanding human-flying fox interactions in the Agusan Marsh Wildlife Sanctuary as basis for conservation policy interventions

- Sherryl L. Paz \& Juan Carlos T. Gonzalez, Pp. 19431-19447

Argentinian odonates (dragonflies and damselflies): current and future distribution and discussion of their conservation

- A. Nava-Bolaños, D.E. Vrech, A.V. Peretti \& A. Córdoba-Aguilar, Pp. 19448-19465

\section{Communications}

The diel activity pattern of small carnivores of Western Ghats, India: a case study at Nelliampathies in Kerala, India

- Devika Sanghamithra \& P.O. Nameer, Pp. 19466-19474

Distribution and threats to Smooth-Coated Otters Lutrogale perspicillata (Mammalia: Carnivora: Mustelidae) in Shuklaphanta National Park, Nepal

- Gopi Krishna Joshi, Rajeev Joshi \& Bishow Poudel, Pp. 19475-19483

Wildlife hunting practices of the Santal and Oraon communities in Rajshahi, Bangladesh - Azizul Islam Barkat, Fahmida Tasnim Liza, Sumaiya Akter, Ashikur Rahman Shome \& M. Fazle Rabbe, Pp. 19484-19491

Ethnozoological use of primates in northeastern India

- Deborah Daolagupu, Nazimur Rahman Talukdar \& Parthankar Choudhury, Pp. 19492-19499

Factors influencing the flush response and flight initiation distance of three owl species in the Andaman Islands

- Shanmugavel Sureshmarimuthu, Santhanakrishnan Babu, Honnavalli Nagaraj Kumara \& Nagaraj Rajeshkumar, Pp. 19500-19508

Birds of Barandabhar Corridor Forest, Chitwan, Nepal

- Saneer Lamichhane, Babu Ram Lamichhane, Kapil Pokharel, Pramod Raj Regmi, Tulasi Prasad Dahal, Santosh Bhattarai, Chiranjibi Prasad Pokheral, Pabitra Gotame,

Trishna Rayamajhi, Ram Chandra Kandel \& Aashish Gurung, Pp. 19509-19526

On some additions to the amphibians of Gunung Inas Forest Reserve, Kedah,

Peninsular Malaysia

- Shahriza Shahrudin, Pp. 19527-19539

Reviews

A review of research on the distribution, ecology, behaviour, and conservation of the Slender Loris Loris lydekkerianus (Mammalia: Primates: Lorisidae) in India

- Mewa Singh, Mridula Singh, Honnavalli N. Kumara, Shanthala Kumar, Smitha D. Gnanaolivu \& Ramamoorthy Sasi, Pp. 19540-19552

Bivalves (Mollusca: Bivalvia) in Malaysian Borneo: status and threats

- Abdulla-Al-Asif, Hadi Hamli, Abu Hena Mustafa Kamal, Mohd Hanafi Idris, Geoffery James Gerusu, Johan Ismail \& Muyassar H. Abualreesh, Pp. 19553-19565

Disentangling earthworm taxonomic stumbling blocks using molecular markers

- Azhar Rashid Lone, Samrendra Singh Thakur, Nalini Tiwari, Olusola B. Sokefun \&

Shweta Yadav, Pp. 19566-19579

A reference of identification keys to plant-parasitic nematodes (Nematoda: Tylenchida) Tylenchomorpha)

- Reza Ghaderi, Manouchehr Hosseinvand \& Ali Eskandari, Pp. 19580-19602

Short Communications

Catalogue of herpetological specimens from Meghalaya, India at the Salim Ali Centre for Ornithology and Natural History

-S.R. Chandramouli, R.S. Naveen, S. Sureshmarimuthu, S. Babu, P.V. Karunakaran \&

Honnavalli N. Kumara, Pp. 19603-19610
A preliminary assessment of odonate diversity along the river Tirthan, Great Himalayan National Park Conservation Area, India with reference to the impact of climate change - Amar Paul Singh, Kritish De, Virendra Prasad Uniyal \& Sambandam Sathyakumar, Pp. 19611-19615

A checklist of orthopteran fauna (Insecta: Orthoptera) with some new records in the cold arid region of Ladakh, India

- M. Ali, M. Kamil Usmani, Hira Naz, Tajamul Hassan Baba \& Mohsin Ali, Pp. 19616-19625

New distribution records of two Begonias to the flora of Bhutan

- Phub Gyeltshen \& Sherab Jamtsho, Pp. 19626-19631

Rediscovery of Aponogeton lakhonensis A. Camus (Aponogetonaceae): a long-lost aquatic plant of India

- Debolina Dey, Shrirang Ramchandra Yadav \& Nilakshee Devi, Pp. 19632-19635

Glyphochloa acuminata (Hack.) Clayton var. laevis (Poaceae): a new variety from central Western Ghats of Karnataka, India

- H.U. Abhijit \& Y.L. Krishnamurthy, Pp. 19636-19639

A cytomorphological investigation of three species of the genus Sonchus L. (Asterales: Asteraceae) from Punjab, India

- M.C. Sidhu \& Rai Singh, Pp. 19640-19644

Dryopteris lunanensis (Dryopteridaceae) - an addition to the pteridophytic diversity of India

- Chhandam Chanda, Christopher Roy Fraser-Jenkins \& Vineet Kumar Rawat, Pp. 1964519648

Notes

First record of Spotted Linsang Prionodon pardicolor (Mammalia: Carnivora:

Prionodontidae) with photographic evidence in Meghalaya, India

- Papori Khatonier \& Adrian Wansaindor Lyngdoh, Pp. 19649-19651

First record of the Eastern Cat Snake Boiga gocool (Gray, 1835) (Squamata: Colubridae) from Tripura, India

- Sumit Nath, Biswajit Singh, Chiranjib Debnath \& Joydeb Majumder, Pp. 19652-19656

First record of the genus Tibetanja (Lepidoptera: Eupterotidae: Janinae) from India - Alka Vaidya \& H. Sankararaman, Pp. 19657-19659

Austroborus cordillerae (Mollusca: Gastropoda) from central Argentina: a rare, little-known land snail

- Sandra Gordillo, Pp. 19660-19662

Intestinal coccidiosis (Apicomplexa: Eimeriidae) in a Himalayan Griffon Vulture Gyps himalayensis

- Vimalraj Padayatchiar Govindan, Parag Madhukar Dhakate \& Ayush Uniyal, Pp. 1966319664

Two new additions to the orchid flora of Assam, India

- Sanswrang Basumatary, Sanjib Baruah \& Lal Ji Singh, Pp. 19665-19670

Wildlife art and illustration - combining black and white ink drawings with colour: some experiments in Auroville, India

- M. Eric Ramanujam \& Joss Brooks, Pp. 19671-19674
Publisher \& Host

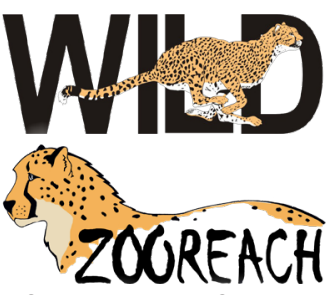

